\title{
Helping Our Patients to Adhere to Chronic Medications: A New Arrow for the Quiver
}

\author{
William H. Shrank, MD, MSHS \\ Division of Pharmacoepidemiology and Pharmacoeconomics, Department of Medicine, Brigham and Women's Hospital and Harvard \\ Medical School, Boston, MA, USA.
}

$\mathrm{J}$ Gen Intern Med 26(12):1394-5

DOI: $10.1007 /$ s1 1606-011-1909-0

(c) Society of General Internal Medicine 2011

I our fragmented healthcare system, doctors need to do more than just prescribe the correct medication for patients. We need to help patients navigate the system and be sure they can purchase what we prescribe.

Non-adherence to chronic medication is a central public health problem, leading to excess morbidity, mortality, and healthcare costs estimated at close to $\$ 300$ billion a year in the U.S. When new prescriptions are written, patients fail to fill them about one quarter of the time. ${ }^{1}$ Virtually every representative body (e.g. World Health Organization, Institute of Medicine, AHRQ, NIH, etc.) in healthcare has identified the reduction of nonadherence as a top priority if we hope to improve healthcare quality and reduce unnecessary costs.

Despite substantial attention to the problem and the implementation of various efforts to reduce nonadherence to chronic medications, we have seen little improvement over time. ${ }^{2}$ This sobering fact is likely a result of the numerous and complex causes of nonadherence to chronic medications, which range from financial barriers, complexity of medical regimens, poor understanding or motivation, adverse cultural beliefs, poor health literacy, and forgetfulness, among others. As a result no single "silver bullet" intervention exists that has been shown to fix the problem of non-adherence. ${ }^{3}$

As a core principle, most would agree that physicians should play an important role in the education, support and motivation of their patients with regard to prescription medication adherence. This has lead to calls for prescribers to better educate their patients about the importance of consistent use of chronic medication therapy, to assess understanding, to engage in a shared-decision making approach, and to motivate patients to take a more active role in their chronic disease management. However, studies of interventions to improve adherence have shown that physicians are not particularly effective at delivering these interventions, raising questions about how to best intervene to encourage appropriate medication use. ${ }^{4}$

But maybe doctors are not emphasizing the correct topics with their patients when it comes to improving medication adherence. It may be that we can be more effective by discussing the actual medication purchasing process and

Published online October 7, 2011 related challenges with each patient. One central challenge for patients in our complex healthcare marketplace is the actual delivery system for medications. Patients take many medications, they receive prescriptions from multiple doctors, they pick them up at multiple pharmacies or mail-order pharmacies, they must travel to retail pharmacies, and they are charged varying copayments for their medications. Just keeping up with all this activity must be challenging for many patients.

Recent studies suggest that if physicians can help a patient identify a low-cost medication from the patient's formulary, their patient's adherence improves. ${ }^{5}$ Other studies suggest that by synchronizing refills on a single day of the month and by consolidating prescription fills in a single pharmacy, adherence tends to be better. ${ }^{6}$ Reducing the number of doses a day is associated with better adherence, as is the use of combination pills to reduce the number of medications taken each day. ${ }^{7}$ Use of pill boxes or other devices to simplify administration can improve adherence, as can simple patient reminders to administer therapy. ${ }^{8}$ In combination, these efforts to simplify prescription drug care may have a meaningful effect on a patient's overall adherence to therapy—and each of these topics can be addressed in a brief conversation between a prescriber and a patient when a new medication is started.

The study from Schmittdiel and colleagues in this issue of JGIM highlights another simple recommendation a prescriber can make. ${ }^{9}$ Patients who use mail-order pharmacies appear to be more adherent to cholesterol medications than those who use retail pharmacies, and the improvement was associated with better cholesterol management. After controlling for patient and prescription characteristics, patients who received a new statin prescription from a mail-order pharmacy were adequately adherent to their statin $87.8 \%$ of the time as compared to $72.9 \%$ of those who used retail pharmacies. Not only did adherence improve, those who used mail-order pharmacies experienced better cholesterol control, with more than $10 \%$ greater rates of meeting ATP-III LDL cholesterol management goals in the mail-order as compared to the retail pharmacy groups.

To address concerns about unmeasured confounding, the authors used both difference-in-differences and instrumental variables approaches, and their findings were robust to both, and to various sensitivity analyses. It should be noted that the study took place in a staff model HMO which may not be representative of other settings, and the observational design used could not demonstrate causation. Nonetheless, the authors used multiple approaches and conducted several sensitivity analyses, and they present convincing evidence that mail-order pharmacy use was associated with 
improved medication-taking behavior in the population they studied.

When considered in the broader context of the literature, the Schmittdiel results can be used to argue for better advice for patients as they administer multiple medications and navigate a complex marketplace. The prescriber must play a central role, and could consider addressing the following issues when prescribing a new medication:

- Cost: Is there a less expensive, but equally effective, medication on the patient's formulary?

- Complexity: Can the number of medications taken daily or the number of daily doses of those medications be reduced?

- Complexity: If the number of medications cannot be reduced, can electronic reminders or improved packaging be employed to support better adherence?

- Channel: Can prescriptions be consolidated and refills synchronized at a single pharmacy or can the use of mail-order pharmacy simplify delivery?

By considering these domains, providers may play an important role in helping patients to overcome these key barriers to appropriate medication adherence.

It may be that pharmacists or representatives from pharmacy benefits managers are poised to assist patients in these conversations. Yet a substantial number of patients never fill a first prescription for a medication, highlighting the important role that the provider must play in helping patients to get started. With the growing emphasis on care coordination and improved primary care, greater collaboration between pharmacy benefits managers, pharmacists and physicians will be instrumental in improving patient behavior.

With implementation of health reform and increasing alignment between provider incentives and improved healthcare quality, doctors have ever more reason to talk to patients about their use of essential chronic therapies and barriers to adherence. Physicians who participate in value-based purchasing, either through Accountable Care Organizations, Patient-Centered Medical Homes, or other mechanisms, will increasingly be responsible for the seamless and efficient management of chronic disease-and adherence to chronic therapies will be a top priority. Greater focus, collaboration and communication around adherence are needed. Schmittdiel and colleagues have provided us with one more tool that we can use to help our patients better care for themselves. Providers should take note, and use this tool wisely.

Corresponding Author: William H. Shrank, MD, MSHS; Division of Pharmacoepidemiology and Pharmacoeconomics, Department of Medicine, Brigham and Women's Hospital and Harvard Medical School, 1620 Tremont Street, Suite 3030, Boston, MA 02120, USA (e-mail: wshrank@partners.org).

\section{REFERENCES}

1. New England Healthcare Institute. Thinking Outside the Pillbox: A Systemwide Approach to Improving Patient Medication Adherence for Chronic Disease. 2009; http://www.nehi.net/publications/44/thinking outside_the_pillbox_a_systemwide_approach_to_improving_patient medication_adherence_for_chronic_disease. Accessed September 20, 2011.

2. Choudhry NK, Setoguchi S, Levin R, Winkelmayer WC, Shrank WH. Trends in adherence to secondary prevention medications in postmyocardial infarction patients. Pharmacoepidemiology and Drug Safety. 2008;17(12):1189-96.

3. Kripalani S, Yao $\mathbf{X}$, Haynes RB. Interventions to enhance medication adherence in chronic medical conditions: a systematic review. Arch Intern Med. 2007;167(6):540-50.

4. Cutrona SL, Choudhry NK, Stedman M, Servi A, Liberman JN Brennan T, Fischer MA, Brookhart MA, Shrank WH. Physician effectiveness in interventions to improve cardiovascular medication adherence: A systematic review. J Gen Intern Med. 2010;25(10):1090-6.

5. Shrank WH, Hoang T, Ettner SL, Glassman PA, Nair K, DeLapp D, Dirstine J, Avorn J, Asch SM. The implications of choice: prescribing generic or preferred pharmaceuticals improves medication adherence for chronic conditions. Arch Intern Med. 2006;166:332-7.

6. Choudhry NK, Fischer MA, Avorn J, Liberman LN, Schneeweiss S, Pakes J, Brennan TA, Shrank WH. The implications of therapeutic complexity on adherence to cardiovascular medications. Arch Intern Med. 2011;171(9):814-22.

7. Claxton AJ, Cramer J, Pierce C. A systematic review of the associations between dose regimens and medication compliance. Clin Ther. 2001;23 (8): 1296-310.

8. Misono AS, Cutrona SL, Choudhry NK, Stedman M, Fischer MA, Liberman JN, Brennan T, Jain SH, Shrank WH. Effectiveness of healthcare information technology (HIT) adherence interventions in cardiovascular disease and diabetes: a systematic review. Am J Manag Care. 2010;16(12 Suppl HIT):SP82-92.

9. Schmittdiel JA, Karter AJ, Dyer W, et al. The comparative effectiveness of mail order pharmacy use vs. local pharmacy use on LDL-C control in new statin users. J Gen Intern Med, 2011; doi:10.1007/s11606-011-1805-7. 